\title{
EDDY EXCHANGE COEFFICIENTS IN NUMERICAL MODELS OF THE PLANETARY BOUNDARY LAYER
}

\author{
C. C. SHIR
}

IBM Research Laboratory, San Jose, Calif., U.S.A.

and

R. D. BORNSTEIN

Dept. of Meteorology, San Jose State University, San Jose, Calif., U.S.A.

(Received 21 June, 1976)

\begin{abstract}
Specification of the eddy exchange coefficients is perhaps one of the most difficuit problems in the numerical modeling of the planetary boundary layer. These coefficients have been computed from finite-difference analogs to analytical expressions associated with surface boundary-layer similarity theory, which is based on observations in an equilibrium surface layer. This procedure leads to erroneous results in the region above the surface layer and in a non-equilibrium surface layer. In addition, differencing problems arise in regions of small vertical wind shear. A new turbulence transport model has been obtained through the closure procedures for the transport equations of the Reynolds stress and the turbulent length scale. The new approach could be used to calculate Reynolds stresses and eddy exchange coefficients throughout a non-neutral planetary boundary layer under non-equilibrium conditions.
\end{abstract}

\section{Introduction}

Specification of the eddy exchange coefficients is one of the most difficult problems associated with modeling the planetary boundary layer. These coefficients have been traditionally computed from finite-difference analogues to various analytical expressions which are associated with surface boundary-layer similarity theory. As the theory is based on observations in an equilibrium surface layer, the procedure could lead to erroneous results in regions above the surface layer, and in a non-equilibrium surface layer over inhomogeneous terrain. In addition, differencing problems can arise in regions of small wind shear, even over homogeneous terrain. This paper summarizes some problems associated with various formulations of the eddy exchange coefficients over homogeneous and inhomogeneous terrain. Results from a one-dimensional numerical planetary boundary-layer model using a $\mathrm{K}$-theory approach are presented to illustrate some of the problems.

A turbulent transport model has been formulated in which the problems mentioned above will not arise, and in which the Reynolds stresses and eddy exchange coefficients are calculated. Shir (1973) used this model to study the turbulent planetary boundary layer under neutral conditions, with results comparing favorably with available data. A simpler form of the model, using the turbulent energy equation, had been used by Shir (1972) to study the air flow over a horizontally inhomogeneous surface. It also yielded realistic results, which were in good agreement with observations. Recently, Lumley and Khajeh-Nouri (1974) 
developed a similar model, in which the closure procedures for the turbulence transport equations were accomplished by functional expansions. However, the effects of wall geometry were not considered, and there were many unknown constants. Wyngaard et al. (1974) used a simplified version of the model to study the atmospheric boundary layer under unstable conditions, with encouraging results. These preliminary studies suggest that the turbulent transport approach can ultimately advance our understanding of turbulent flows in the atmosphere.

\section{Problems With $K$-Theory over Homogeneous Terrain}

This section discusses some of the problems associated with the specification of the eddy exchange coefficients $K(z)$ in a one-dimensional numerical boundarylayer model over homogeneous terrain. Additional problems associated with twoand three-dimensional models over inhomogenous terrain are discussed in the following section.

Stevens (1959) was one of the first to incorporate the effects of the "local stability' at each level on the values of $K(z)$ in the planetary boundary layer by use of the following expression

$$
K(z)=\frac{K(h)[1-n(z-h)]}{1+\alpha \partial \theta / \partial z},
$$

where $\theta$ is the potential temperature, $h$ is the height of the surface boundary layer, and $\alpha, n$, and $K(h)$ are prescribed constants. A similar approach was tried by Fisher and Capian (1963), who used

$$
K(z)=K^{*}(z) \exp [-\alpha(z) \partial \theta / \partial z],
$$

where $\alpha(z)$ and $K^{*}(z)$, the expected eddy coefficients under neutral conditions, were specified.

Estoque (1961) introduced a surface boundary layer based on similarity theory, into a numerical planetary boundary-layer model. This allowed the mixing coefficients in the upper numerical transition layer to be dependent on the structure of the surface layer, via the computed values of $K(h)$, as follows,

$$
K(z)=K(h)(H-z) /(H-h), \quad z>h,
$$

where $H$ is the upper boundary of the transition layer. Values of $K(h)$ were computed from

$$
K(z)=l^{2}\left|\frac{\partial V}{\partial z}\right|\left[\phi_{m}(\mathrm{Ri})\right]^{-2}, \quad z \leq h,
$$

where $l$ is the Prandtl mixing length, given as $k z$, with $k$ as the von Kármán constant, $V$ the horizontal wind speed, and $\phi_{m}$ the non-dimensional wind shear, or stability function, which has a value of unity in a neutral, equilibrium surface boundary layer. This function is dependent on the local Richardson number Ri, 
which is given by

$$
\mathrm{Ri}=\frac{g}{\theta_{m}} \frac{\partial \theta / \partial z}{(\partial V / \partial z)^{2}},
$$

where $g$ is the acceleration due to gravity, and $\theta_{m}$ is the space-averaged potential temperature. Note that values of $K(z)$ in the numerical transition layer obtained from (3) are not dependent on local gradients, as were those obtained from (1) or (2).

A new approach was tried by Pandolfo et al. $(1963,1965)$, in which $K(z)$ in the entire boundary layer was again made dependent on local stability by the application of (4) to heights up to several kilometers. In applying that equation, the following expression (from Blackadar, 1962) was used for the mixing length $l$ in the transition layer

$$
l=\frac{k z}{1+k z / \lambda},
$$

where $\lambda$ is the value of $l$ in the free atmosphere, in turn dependent on the geostrophic wind speed. They used the following forms for the stability function $\phi_{m}$ : the Monin-Obukhov (1954) form for forced convection, and the Priestley (1959) form for free convection. However, a significant number of investigators using this or similar formulations for $K(z)$, i.e., those dependent on local values of $\mathrm{Ri}$, report the appearance of a local minimum value of $K$ at a height of several hundred meters, with geostrophic wind speeds less than about $8 \mathrm{~m} \mathrm{~s}^{-1}$.

During previously unpublished preliminary experiments with a one-dimensional version (over homogeneous terrain) of the two-dimensional URBMET urban boundary layer, Bornstein (1972) also used Equations (4) through (6). The initial profiles of wind, potential temperature and eddy exchange coefficients; for a typical case, as well as those after six hours of simulated time (at 2400 LST), are shown in Figure 1. The wind profile at 2400 LST shows a weak low-level jet, i.e.,
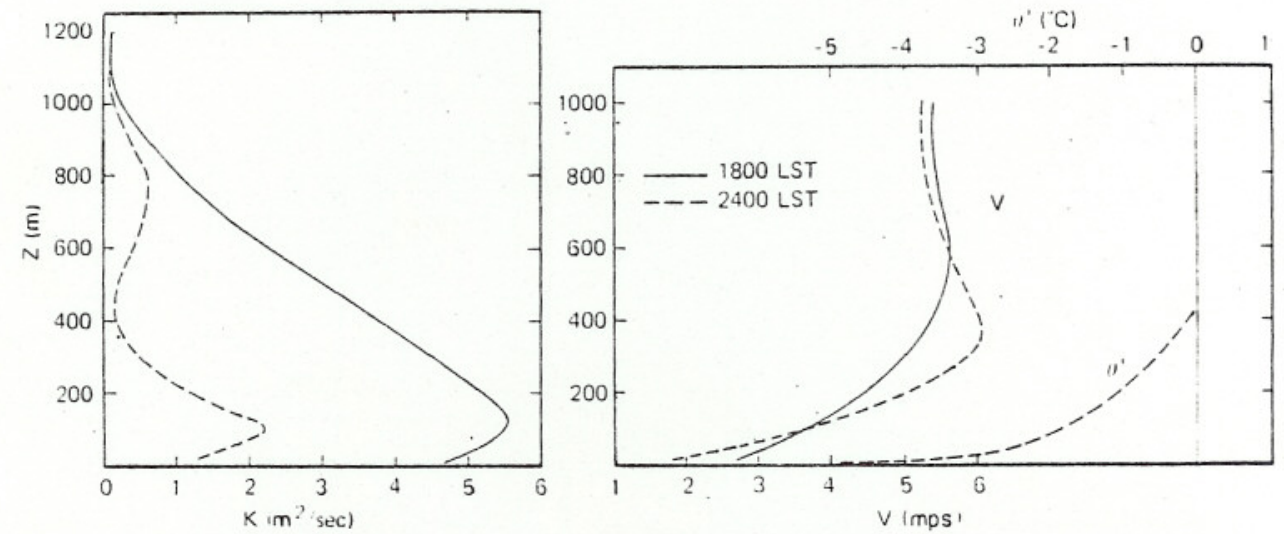

Fig. 1. Vertical distributions of eddy coefficients $K$, wind speed $V$, and perturbation potential temperature $\theta^{\prime}$ at initial time of 1800 LST and after six hours of simulated time. 
slightly supergeostrophic speeds, at a height of several hundred meters, while the corresponding $K(z)$ profile indicates a 'local minimum' at that level. The corresponding profile of potential temperature shows that the effects of surface cooling have been confined to the layer below the local minimum.

The impediment of the downward flow of heat, which results from such a local minimum in this and other models, produces surface temperatures which are lower than those actually observed, e.g., see the results of Pandolfo et al. (1963), Wu (1965), Zdundowski et al. (1967), Tag (1969), Luther (1969), and Sasamori (1970). In addition, the same effect produced urban circulation cells in the preliminary two-dimensional version of URBMET (unpublished), which were confined to the layer below the local minimum. With a different formulation for $K(z)$, discussed below, the local. minimum was eliminated, and the depth of the circulation cells increased to a more realistic value.

The local minimum in the $K$ profiles arises when the finite-difference analog to $\mathrm{Ri}$ is evaluated in the region of a nocturnal low-level jet. A layer of small vertical wind shear in the region of the jet results in a large value of the "finitedifference" Richardson number $\mathrm{Ri}_{\Delta}$ when it is computed by the numerical analog to (5) given below

$$
\mathrm{Ri}_{\Delta}=\frac{g}{\theta_{m}} \frac{\theta_{2}-\theta_{1}}{\left(V_{2}-V_{1}\right)^{2}}\left(z_{2}-z_{1}\right) .
$$

The large value leads to a large value of $\phi_{m}(\mathrm{Ri})$, and hence a small value of $K$, when the following Monin-Obukhov form (for a stable layer) is used for $\phi_{m}$

$$
\phi_{m}=(1-\alpha \mathrm{Ri})^{-1} .
$$

However, Lile (1970) has observed that the largest values of $K$ within the elevated west-coast subsidence inversion occur in regions near the wind maxima. His eddy viscosity was estimated from tetroon-derived observations of the perturbation velocities.

Thus, the values of $\mathrm{Ri}_{\Delta}$ give valid estimates of atmospheric stability only in layers of strong wind shear, e.g., in the surface boundary layer. Observations by Lyons et al. (1964) have shown that when values of $\mathrm{Ri}_{\Delta}$ were calculated from real data at heights of about one hundred metres, with small wind shears and near-adiabatic lapse rates, the results were "quite imprecise".

The value of the "constant" $\alpha$ in (8) is related to the critical Richardson number $\mathrm{Ri}_{c}$ (above which turbulence tends to be suppressed) by

$$
\frac{1}{\alpha}=\mathrm{Ri}_{\mathrm{c}}>0 \text {. }
$$

Combining (4) and (8) shows that $\mathrm{Ri}_{c}$ is the maximum allowable value of $\mathrm{Ri}$ in the Monin-Obukhov formulation. However, the correct value of $\mathrm{Ri}_{c}$ is uncertain; for example, Crawford (1965), Panofsky and Prasad (1965), and Reiter and Lester (1967) have shown that values of $\mathrm{Ri}_{\Delta}$ and $\mathrm{Ri}_{c}$ must increase with layer 
thickness. In addition, Lyons et al. (1964) have concluded that "it is quite possible that there is no unique value of $\mathrm{Ri}_{c}$, and that $\mathrm{Ri}_{\mathrm{c}}$ will increase with increasing height." Zdundowski et al. (1967) and Oke (1970a) have echoed this thought.

If $\mathrm{Ri}_{c}$ does change with height, then from (9), $\alpha$ also changes with height. Observations in stable layers, within $8 \mathrm{~m}$ of the surface, yield values of $\alpha$ ranging from 7 (McVehil, 1964) to 0.3 (Neumann and Mahrer, 1971). Businger et al. (1971) have stated that there are "still not enough data for substantiation" of a particular value, although the latest estimates are close to 5, e.g., those of Oke (1970a, 1970b), Webb (1970), and Okamoto and Webb (1970).

Other observations have shown that $\alpha$ is a function of stability; for example, O'Brien (1965) found it equal to 7.5 for slight inversions, but equal to 3.5 for extreme inversions. Panofsky et al. (1960) have found that the original value of 0.6, proposed by Monin and Obukhov (1954), gave a better fit to profile data over a wider range of stability, than did a value of 4.5 , which fit best over a narrower range. Similar results were obtained by Yamamoto (1959) and Takeuchi (1961), while Lyons et al. (1964) report that $\alpha$ could be less than unity.

The values of $\alpha$ used in numerical planetary boundary-layer models have generally been smaller than those derived from tower observations, because the relatively large grid spacing used in the models, i.e., 25 to $100 \mathrm{~m}$, yields large values of $\mathrm{Ri}_{\Delta}$. In addition, the low speeds, and hence, the small vertical shears, especially in models of a sea breeze arising in otherwise calm conditions, also lead to large values of $\mathrm{Ri}_{\Delta}$. Hence, McPherson (1970) used a value of 0.03 for $\alpha$, Estoque (1961) used 1.0, and Neumann and Mahrer (1971) used 0.3; Zdundowski et al. (1967) used a, value dependent on height, viz., 7 below $10 \mathrm{~m}, 1.25$ above $100 \mathrm{~m}$, and interpolated values inbetween. Various other methods have been employed in numerical models when $\mathrm{Ri}_{\Delta}$ becomes too large, and hence $K(z)$ too small; for example, Tag (1969) divided his values by 5 , equivalent to reducing $\alpha$ from 3 to 0.6 . Estoque and Bhumralkar (1968) used a stability parameter $S$ given by

$$
S=\frac{\sqrt{g}}{\theta_{m}} \frac{\partial \theta / \partial z}{\partial V / \partial z},
$$

in which wind shear appears to the first power and not to the second, as in (5). On the other hand, Pandolfo et al. (1963) introduced a minimum value for $K$ of $10^{4} \mathrm{~cm}^{2} \mathrm{~s}^{-1}$, while Estoque and Bhumralkar (1969) used a value of $\mathrm{Ri}$ for the entire boundary layer which, while averaged through the lowest $100 \mathrm{~m}$, was not dependent on the local stability at other levels.

Sasamori (1970) overcame the problem by using a third-order polynomial developed by O'Brien (1970), which increased the magnitude of the computed $K$-values at $200 \mathrm{~m}$ from $10^{3}$ to $5 \times 10^{4} \mathrm{~cm}^{2} \mathrm{~s}^{-1}$, and wiped out an erroneously predicted surface temperature inversion. This formulation, also used by Bornstein $(1972,1975)$ in the final version of URBMET, assumes that the following are 
known:

$$
K\left(H^{*}\right), \quad(\partial K / \partial z)_{H *}, \quad K(h), \quad(\partial K / \partial z)_{h},
$$

where $H^{*}$ is the level at which $K$ has decreased to some small value, not necessarily the top of the transition layer. If the vertical variation of $K$ at $H^{*}$ is taken as zero, then $K(z)$ is given by

$$
\begin{aligned}
& K(z)=K\left(H^{*}\right)+\left(\frac{Z-H^{*}}{H^{*}-h}\right)^{2}\left\{K(h)-K\left(H^{*}\right)\right. \\
& \left.+(z-h)\left[\left(\frac{\partial K}{\partial z}\right)_{h}+2 \frac{\left[K(h)-K\left(H^{*}\right)\right]}{H^{*}-h}\right]\right\} .
\end{aligned}
$$

The equation yields parabolic curves under all stabilities, as predicted by Blackadar (1962), Lettau (1962), and Lettau and Dabberdt (1970), and as observed during night-time conditions by Elliot (1964). Deficiencies in this formulation include the prescribed shape and value of $H^{*}$, and the lack of dependence of $K$ on local stability.

The assumption concerning the shape of the vertical distribution of $K$ described above is somewhat arbitrary. The following vertical distribution of $K$ under neutral conditions best fits the results from the one-dimensional version of the turbulence transport model of Shir (1973):

$$
\tilde{K}=k \tilde{z} \exp (-4 \tilde{z})
$$

where $\tilde{K}$, the dimensionless eddy coefficient, is related to $K$, the dimensional eddy coefficient, such that

$$
K=u_{*} H \tilde{K} \text {. }
$$

In addition, $z$ the dimensionless height is related to $z$ by

$$
z=\tilde{z} H,
$$

where

$$
H=0.5 u_{*} / f
$$

where $u_{*}$ is the friction (scaling) velocity and $f$ is the Coriolis parameter. Note that (11a) is similar in form to that recommended by Arya (1973).

Interestingly, Businger and Arya (1974) assumed that the following form for $K$ is valid during neutral and stable conditions:

$$
\tilde{K}=\frac{k \tilde{z}}{1+\alpha \phi_{m}} \exp \left(-\left|\frac{V_{g}}{2 u_{*}}\right| \tilde{z}\right),
$$

where $V_{g}$ is the geostrophic wind component orthogonal to the surface wind. Note that if $V_{\mathrm{g}}$ is approximately $8 u_{*}$, as has been predicted during neutral conditions by the turbulence transport models of Wyngaard (1973) and Deardorff (1970), then both (11a) and (11b) are very similar. Lamb et al. (1974) calculated 
the eddy diffusivity of virtual particles by employing a sub-grid turbulence model. They also found that the optimal eddy diffusivity under neutral conditions is very close to that described by Equation (11a).

The value of the local Richardson number may not always give a true estimate of the total eddy mixing process under extremely unstable conditions. This is because the unstable surface boundary layer is frequently capped by a nearneutral Ekman layer, as shown by Kuo (1968). Thus, the effect on the Ekman layer of thermals originating in the surface boundary layer would not be modeled by an Ekman-layer formulation for $K(z)$ which is dependent on local gradients.

These thermals are too large to be modeled by the diffusion term and too small to be simulated by the vertical advection term. Observational evidence for active convection during near-neutral conditions aloft was presented by Ackerman and Appleman (1975), while the "penetrative convection" term of Estoque and Bhumralkar (1969) was án attempt to parameterize the Cumulus convection process in a numerical boundary-layer model. Thus, an Ekman layer formulation for $K(z)$ which is dependent only on the gradients in the surface boundary layer might better represent the total effect of the eddy process in the Ekman layer under extremely unstable conditions. This is consistent with the observations of Kuo (1968) who showed that the value of $K$ at $z$ in the Ekman layer at O'Neill depended "not only on the local value of $\partial \theta / \partial z$, but also on the integrated stability, from the surface level up to the equilibrium level; that is, to the level where $\theta$ equals the surface value $\theta_{s}: "$

Thus, while standard approaches for specifying $K(z)$ over homogeneous terrain appear to be adequate under neutral conditions, there is no completely acceptable formulation for describing them over the entire range of non-neutral conditions.

\section{Problems With $K$-Theory over Non-Homogeneous Terrain}

The previous section presented some of the problems that arise with formulations for $K(z)$, that have been used in numerical models of the equilibrium boundary layer over homogeneous terrain. This section will discuss some of the additional problems arising in layers over non-homogeneoús terrain. In regions a and $\mathrm{d}$ of Figure 2, the values of $K(z)$ are obviously related to the surface roughnesses $z_{0}^{\prime}$ and $z_{0}$, respectively, while in region $\mathrm{c}$ they are related to both roughnesses. A following section will demonstrate that $K(z)$ in region b is related to $z_{0}^{\prime}$, and not $z_{0}$, and that the value of $\phi_{m}$ in. Equation (4) in a neutral non-equilibrium surface boundary layer over heterogeneous terrain (region c) is not equal to unity; as it is over homogeneous terrain. It is also not equal to unity in a neutral, equilibrium planetary boundary layer over homogeneous terrain, as will also be shown in a following section.

As Peterson (1971) has pointed out, the profile $K(z)$ over heterogeneous terrain must depend on horizontal, as well as vertical, gradients. For neutral 




Fig. 2. Configuration of the flow over an inhomogeneous surface, in which the development of the equilibrium boundary layer EBL and internal boundary layer IBL is shown.

stability, Heisenberg (1948) reasoned from turbulence theory that

$$
K=\varepsilon^{1 / 3} l^{4 / 3},
$$

where $\varepsilon$ is the rate of dissipation of turbulent energy per unit mass, given by

$$
\varepsilon=K S^{2} \text {. }
$$

The quantity $S$ is related to the divergence and deformation of the flow by the following,

$$
S=\left[2\left(\frac{\partial u_{i}}{\partial x_{i}}\right)^{2}+\left(\frac{\partial u_{j}}{\partial x_{k}}+\frac{\partial u_{k}}{\partial x_{j}}\right)^{2}\right]^{1 / 2}
$$

where the first term is the sum of three terms, and the last set of parentheses includes nine terms.

Combining (12) and (13) yields the well-known expression

$$
K=l^{2} S \text {. }
$$

For a boundary layer over homogeneous terrain, (14) simplifies to the form for the wind shear appearing in (4), i.e.,

$$
S=\left[\left(\frac{\partial u}{\partial z}\right)^{2}+\left(\frac{\partial v}{\partial z}\right)^{2}\right]^{1 / 2}
$$

For a two-dimensional, incompressible, slab-symmetric boundary layer, e.g., like that in the URBMET model of Bornstein $(1972,1975)$, (14) becomes

$$
S=\left[2\left(\frac{\partial u}{\partial x}\right)^{2}+2\left(\frac{\partial w}{\partial z}\right)^{2}+\left(\frac{\partial v}{\partial z}\right)^{2}+\left(\frac{\partial v}{\partial x}\right)^{2}+\left(\frac{\partial u}{\partial z}+\frac{\partial w}{\partial x}\right)^{2}\right]^{1 / 2} .
$$


The URBMET model is currently being used to simulate the flow of a neutral planetary boundary layer over a discontinuity in surface roughness, and comparative results from using both (16) and (17), will be available in the near future.

\section{Turbulence Transport Model}

The fast growing capabilities of the high-speed computer enables the exploration of the possible application of high-order turbulence transport models to planetary boundary-layer problems. A turbulence transport model for a neutral atmospheric boundary layer was described in detail by Shir (1973). This model includes equations which describe the dynamics of the Reynolds stresses, turbulence length scale, and mean wind. Results from this model duplicated many interesting aspects of the atmospheric boundary layer, in particular, the vertical distribution of the eddy coefficients.

\section{A. Vertical distribution of the eddy Coefficient above the surface layer}

The eddy coefficient, the ratio of the shear stress to the mean wind shear, was calculated from computed values of these two quantities, and its vertical distribution is shown in Figure 3. Values equal $k \tilde{z}$ near the surface and reach a maximum



Fig. 3. Vertical distribution of computed non-dimensional eddy diffusivity coefficients and empirical formulas for the distribution. 
near $\tilde{z}=0.3$. It is of practical use in planetary-layer modeling to prescribe a simple form for the vertical variation of the eddy coefficient. Equation (11a) is a simple formula, which fits the predicted profile for $\tilde{z} \leq 0.4$, but as shown in the Figure, it decreases too rapidly above this level. The following more complex form

$$
K=\frac{k \vec{z}}{2}\left[e^{-4 \bar{z}}+\frac{1}{16 \tilde{z}^{1.6}}\right]
$$

fits the computed profile well throughout the entire layer.

Either Equation (11a), or (18) could be used for modeling neutral conditions. However, the profile of $K$ under non-neutral conditions has not yet been fully investigated with the present turbulent transport model. The model could be extended to non-neutral conditions by including the equations which govern the mean temperature, temperature-velocity correlations, temperature variance, and temperature time scale.

The non-dimensional wind shear $\phi_{m}$ was calculated, using the turbulent transfer model of Shir (1973), herein referred to as S3, from the following equation

$$
\phi_{m}=\frac{k z}{u_{*}} \frac{\partial V}{\partial z}
$$

where the constant friction velocity $u_{*}$ is a specified scaling velocity, and the wind shear is given by

$$
\frac{\partial V}{\partial z}=\sqrt{\left(\frac{\partial u}{\partial z}\right)^{2}+\left(\frac{\partial v}{\partial z}\right)^{2}}
$$

The following equations from S3 were used to evaluate the two gradients on the right side of (20)

$$
\begin{aligned}
& \frac{\partial}{\partial t}\left(\frac{\partial u}{\partial z}\right)=f \frac{\partial v}{\partial z}-\frac{\partial^{2} \overline{u^{\prime} w^{\prime}}}{\partial z^{2}} \\
& \frac{\partial}{\partial t}\left(\frac{\partial v}{\partial z}\right)=-f \frac{\partial u}{\partial z}-\frac{\partial^{2} \overline{v^{\prime} w^{\prime}}}{\partial z^{2}}
\end{aligned}
$$

where $\overline{u^{\prime} w^{\prime}}$ and $\overline{v^{\prime} w^{\prime}}$ are given by $(11 \mathrm{~g})$ and $(11 \mathrm{~h})$ in $\mathrm{S} 3$, respectively.

The vertical distribution of $\phi_{m}$ in a neutral planetary boundary layer over a homogeneous surface, computed from the above equations, is shown in Figure 4. Its value increased from unity at the surface to a maximum of 1.6 in the middle of the planetary boundary layer, and then it decreased to a small value at the top of the layer.

Thus, even under neutral conditions, large errors will result if $\phi_{m}$ in (19) is assumed to have a value of unity above the surface boundary layer. This results as the mixing length above the surface boundary layer is not given by $k z$ and because $u_{*}$ is not constant above the surface boundary layer. 


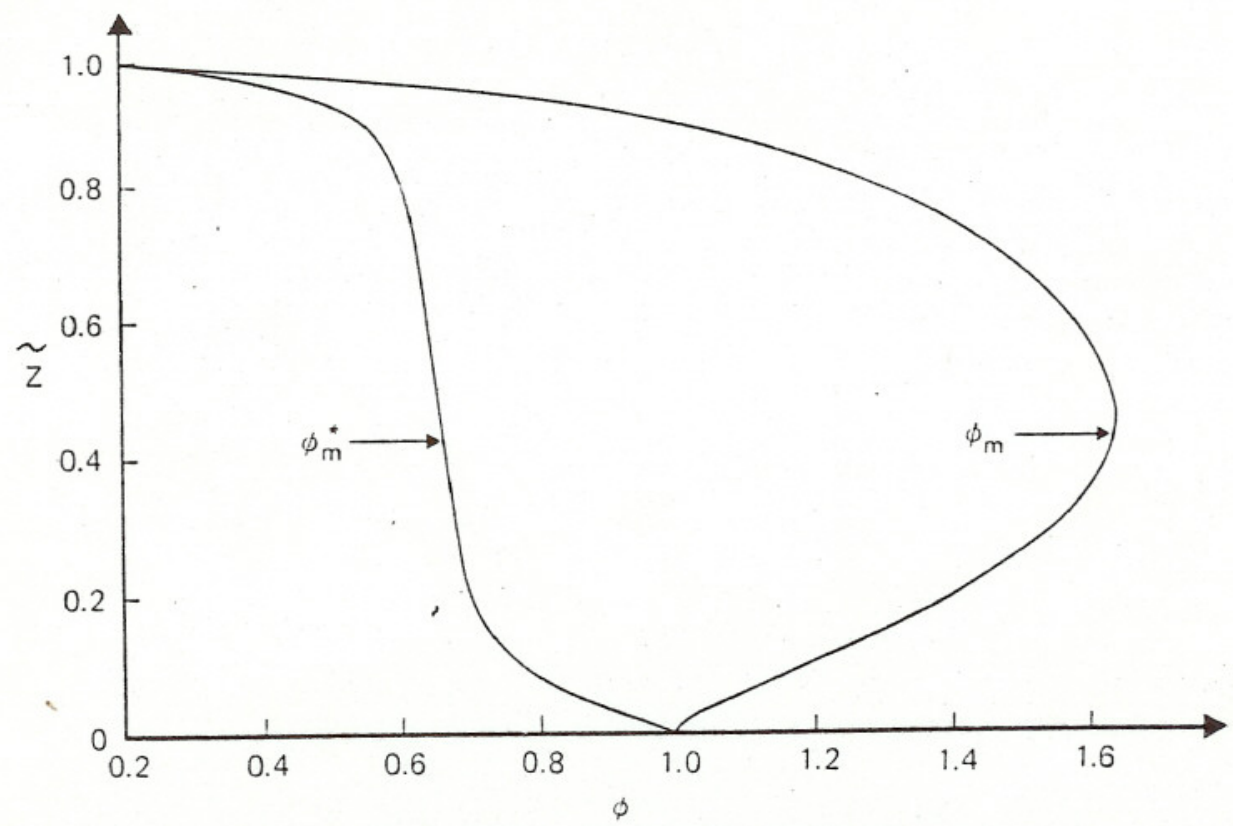

Fig. 4. Vertical distribution of two forms of computed non-dimensional wind shear.

The following more general form for the $\phi_{m}$ in (19) does not make any assumptions about the mixing length or the stress, and is frequently used in the modeling of a neutral planetary boundary layer over homogeneous terrain

$$
\phi_{m}^{*}=\frac{l}{\sqrt{\tau / \rho_{m}}}\left|\frac{\partial V}{\partial z}\right|
$$

where $\rho_{m}$ is the average density in the boundary layer, $l$ is given by (6), and $\tau$ is given by

$$
\frac{\tau}{\rho_{m}}=K\left|\frac{\partial V}{\partial z}\right|
$$

Note that combining (23) and (24) yields Equation (4).

In the $\mathrm{S} 3$ model, $l$ was evaluated from its (11i),.which was derived from

$$
l=\frac{c q^{3}}{\varepsilon},
$$

where $q$ is the square root of the specific turbulent energy, evaluated from (11j) in S3, $\varepsilon$ is its rate of dissipation, and $c$ is a constant.

Excellent agreement was shown in Figure 5 of S3 between predicted values of $l$ and those observed by Blackadar (1962). In the $\mathrm{S} 3$ model, $\tau$ is computed from

$$
\frac{\tau}{\rho_{m}}=\left(\overline{u^{\prime} w^{\prime}}\right)^{2}+\left(\overline{v^{\prime} w^{\prime}}\right)^{2}
$$




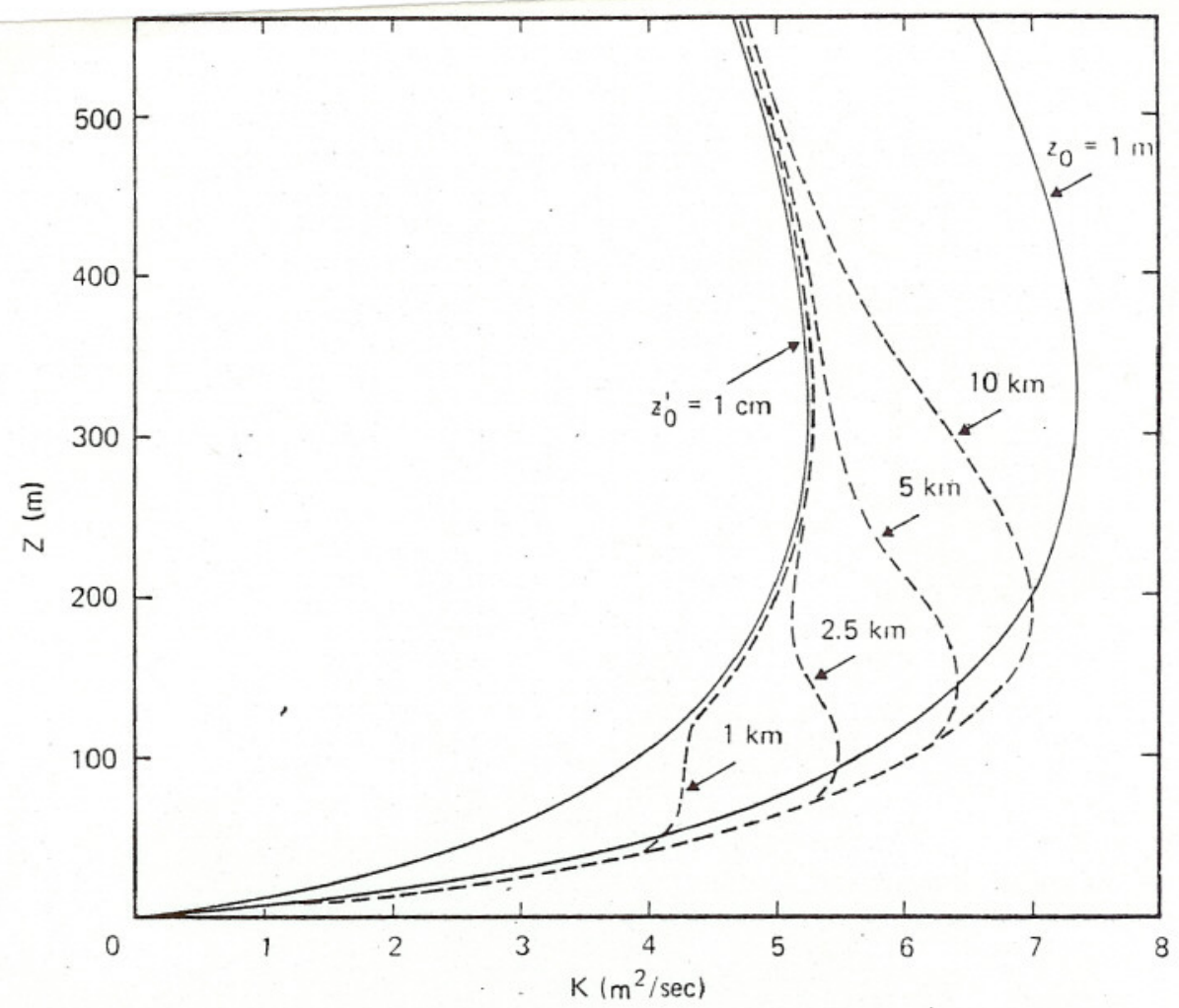

Fig. 5. Vertical distribution of computed eddy diffusivity coefficients over inhomogeneous terrain at various downwind fetch distances. Also shown are the equilibrium profiles for the two surface roughnesses.

Values of $\phi_{m}^{*}$ (Figure 4) evaluated from (20), (25) and (26) show that it, like $\phi_{m}$, is not unity in a neutral boundary layer over homogeneous terrain. Its value decreased from unity at the surface to 0.65 at the middle of the boundary layer, and then to a small value at its top.

\section{B. EFFECTS OF HORIZONTAL INHOMOGENEITY}

When air flows over inhomogeneous terrain, e.g., over a change of surface roughness or surface temperature, there exists a transition region (region $\mathrm{c}$ in Figure 2), in which the turbulence is not in equilibrium, but in which it is still adjusting to the new surface condition. Results from Shir (1972) indicate that the adjusting process is rather slow, implying that the transition region occupies a significant portion of the area above the new surface. In the transition region, the eddy coefficients are not only a function of height, but also a function of the downwind fetch from the edge of the change, as well as of both surface conditions.

The vertical distribution of $K$ at various distances downwind from a change in surface roughness (from $1 \mathrm{~cm}$ to $1 \mathrm{~m}$ ) is shown in Figure 5 . The change in the $K$-profile starts near the surface and then gradually propagates upward. Values 
mrst oversnool, and men approacn mose corresponaing to tne new surrace. in mis case, it takes a distance of more than $10 \mathrm{~km}$ for the $K$-profiles to adjust completely to the new surface conditions. Hence, if the $K$-profiles are assumed to adjust to the new surface conditions immediately, their values in the transition region will be overestimated for the case of a rough-to-smooth change and underestimated for the case of a smooth-to-rough change.

\section{Conclusions}

Formulations for the eddy exchange coefficients $K(z)$ which are dependent on numerical analogues to the finite-difference Richardson number $\mathrm{Ri}_{\Delta}$ are only valid in layers of monotonic wind speed changes. Thus, in a stable layer, at low wind speed, the values of $\mathrm{Ri}_{\Delta}$ become very large in the region of the night-time low-level jet. This produces a minimum value of. $K(z)$ in the very region where observations have shown large values. In addition, this local minimum impedes the predicted downward flow of heat and momentum, thus confining the effects of night-time surface cooling to the layer below the local minimum.

Formulations in which the $K$-profiles are not dependent on local gradients, and/or have a prescribed shape, are limited in that they cannot treat changes in stability with height, as found, for example, in the case of an elevated subsidence inversion. However, the effect on the Ekman layer of thermals originating in the surface boundary layer would not be modeled by an Ekman-layer formulation for $K(z)$ which is dependent on local gradients. Thus an Ekman-layer formulation which is dependent only on the gradients in the surface boundary layer might better represent the total effect of the eddy process in the Ekman layer under extremely unstable conditions.

The value of the critical finite-difference Richardson number $\mathrm{Ri}_{c}$ (for the transition to turbulent flow) appears to depend on the thickness of the layer through which it is evaluated. Numerical models of the planetary boundary layer, which use relatively large vertical grid spacings ( 25 to $100 \mathrm{~m}$ ), have correctly used values of $\mathrm{Ri}_{c}$ which tend to be larger than those computed from observations on relatively short towers.

Formulations for the eddy coefficients over non-homogeneous terrain that do not consider non-equilibrium conditions, horizontal gradients, and the "history" of the flow cannot yield entirely accurate results. Such conditions have begun to be incorporated into new turbulence transport models which will advance our understanding of the turbulent planetary boundary layer.

\section{Acknowledgements}

The authors thank the personnel at the IBM Research Laboratory at San Jose, California, and at the IBM Scientific Center at Palo Alto, California, for their interest and assistance. Thanks are also extended to Mrs Linda LaDuca for the typing of this manuscript. 


\section{References}

Ackerman, B. and Appleman, H.: 1975, Thermodynamic Structure Across a City on Days with Convective Rains, Presented at 55th Annual Meeting. American Meteorological Society, Denver.

Arya, S. P. S.: 1973, 'Neutral Planetary Boundary Layer Above a Non-Homogeneous Surface', Geophys. Fluid. Dyn. 4, 333-355.

Blackadar, A. K.: 1962, 'The Vertical Distribution of Wind and Turbulent Exchange in a Neutral Atmosphere', J. Geophys. Res. 67, 3095-3102.

Bornstein, R. D.: 1972, 'Two Dimensional, Non-Steady Numerical Simulations of Nighttime Flows of a Stable Planetary Boundary Layer over a Rough Warm City', Ph.D. Thesis, Dept. of Meteorology and Oceanography, New York University. Available from University Microfilm, Inc.

Bornstein, R. D.: 1975, 'The Two Dimensional URBMET Urban Boundary Layer Model', J. Appl. Meteorol., 14, 1459-1477.

Businger, J. A. and Arya, S. P. S.: 1974, 'Height of the Mixed Layer in the Stably Stratified Planetary Boundary Layer', Adv. Geophys. 18A, 73-92.

Businger, J. A., Wyngaard, J. C., Izumi, Y., and Bradley, E. F.: 1971, 'Flux Profile Relationships in the Atmospheric Surface Layer', J. Atmos. Sci. 28, 181-189.

Crawford, T. V.: 1965, 'Moisture Transfer in Free and Forced Convection', Quart. J. Roy. Meteorol. Soc. 75, 18-27.

Deardorff, J. W.: 1970, 'Preliminary Results from Numerical Integrations of the Unstable Planetary Boundary Layer', J. Atmos. Sci. 27, 1209-1211.

Elliott, W. P.: 1964, 'The Height Variation of Vertical Heat Flux Near the Ground', Quart. J. Roy. Meteorol. Soc. 90, 260-265.

Estoque, M. A.: 1961, 'Theoretical Investigation of the Sea Breeze', Quart. J. Roy. Meteorol. Soc. 87, 136-146.

Estoque, M. A. and Bhumralkar, C. M.: 1968, 'Theoretical Studies of the Atmospheric Boundary Layer', Res. and Dev. Tech. Rep., ECOM-G7G2-F, 46 pp.

Estoque, M. A. and Bhumralkar, C. M.: 1969, 'Further Studies on Flow over Non-Homogeneous Terrain', Tech. Rep. Div. of Atmos. Sci., Univ. of Miami.

Fisher, E. L. and Caplan, P.: 1963, 'An Experiment in Numerical Prediction of Fog and Stratus', J. Atmos. Sci. 20, 425-437.

Heisenberg, W.: 1948, 'Zur statistischen Theorie der Turbulenz', Z. Physik 124, 628-657.

Kuo, H. L.: 1968, 'The Thermal Interaction Between the Atmosphere and the Earth and Propagation of Diurnal Temperature Waves', J. Atmos. Sci. 25, 682-706.

Lamb, R. G., Chen, W. H., and Seinfeld, J. H.: 1974, 'Numerico-Empirical Analyses of Atmospheric Diffusion Theories', Symposium on Atmos. Diff. and Air Pollution, Santa Barbara, Calif., Sept., 1974.

Lettau, H. H.: 1962, 'Theoretical Wind Spirals in the Boundary Layer of a Barotropic Atmosphere', Beitr. Phys. Atmos. 35, 195-212.

Lettau, H. H. and Dabberdt, W. F.: 1970, 'Variangular Wind Spirais', Boundary-Layer Meteorol. 1, 64-79.

Lile, R. C.: 1970, 'Tetroon-Derived Eddy Viscosity in the Region of the Elevated West Coast Temperature Inversion', San Jose State University Report No. 1, San Jose, Calif.

Luther, F. M.: 1969, 'A Numerical Model of the Energy Transfer Processes in the Lower Atmosphere', Ph.D. Thesis, Univ. of Calif., Davis.

Lumley, J. L. and Khajeh-Nouri, B.: 1974, 'Computational Modeling of Turbulent Transport', Adv. Geophys. 18A, 169-192.

Lyons, R., Panofsky, H. A., and Wollaston, S.: 1964, 'The Critical Richardson Number and its Implications for Forecast Problems', J. Appl. Meteorol. 3, 136-142.

McPherson, R. D.: 1970, 'A Numerical Study of the Effect of a Coastal Irregularity on the Sea Breeze', J. Appl: Meteorol. 9, 767-777.

McVehil, A.: 1964, 'Wind and Temperature Profiles near the Ground in Stable Stratification', Quart. J. Roy. Meteorol. Soc. 90, 136-146.

Monin, A. S. and Obukhov, A. M.: 1954, 'Basic Regularity in Turbulent Mixing in the Surface Layer of the Atmosphere', U.S.S.R. Acad. Sci. Geophys. Inst. No. 24. 
Neumann, J. and Mahrer, Y.: 1971, 'A Theoretical Study of the Land and Sea Breeze Circulations', J. Atmos. Sci. 28, 532-542.

O'Brien, J.: 1965, 'An Investigation of the Adiabatic Wind Profile of the Atmospheric Boundary Layer', J. Geophys. Res. 70, 2277-2290.

O'Brien, J.: 1970, 'On the Vertical Structure of the Eddy Exchange Coefficient in the Planetary Boundary Layer', J. Atmos. Sci. 27, 1213-1215.

Okamoto, M. and Webb, E. K.: 1970, 'The Temperature Fluctuations in Stable Stratification', Quart. J. Roy. Meteorol. Soc. 96, 591-600.

Oke, T. R.: 1970a, 'Turbulent Transport Near the Ground in Stable Conditions', J. Appl. Meteorol. 9, 778-785.

Oke, T. R.: 1970b, 'The Temperature Profile Near the Ground on Calm Clear Nights', Quart. J. Roy. Meteorol. Soc. 96, 14-23.

Pandolfo, J., Cooley, D., and Newburg, E.: 1963, 'Preliminary Investigations of Numerical Models for the Short-Period Prediction of Wind, Temperature, and Moisture in the Atmospheric Boundary Layer', Final Rept. 7047-80, The Travelers Research Center, Inc.

Pandolfo, J. and Atwater, M. A.: 1965, 'The Development of a Numerical Prediction Model for the Planetary Boundary Layer', Final Rept. 7465-174, Contract Cwb-10960, The Travelers Research Center, Inc.

Panofsky, H. A., Blackadar, A. K., and McVehil, G. E.: 1960, 'The Adiabatic Wind Profile', Quart. J. Roy. Meteorol. Soc. 86, 390-398.

Panofsky, H. A. and Prasad, B.: 1965, 'Similarity Theories and Diffusion', Inter. J. Air Water Poll. 9, $419-430$.

Peterson, E. W.: 1971, 'Predictions of the Momentum Exchange Coefficient for Flow over Heterogeneous Terrain', J. Appl. Meteorol. 10, 958-961.

Priestley, C. H. B.: 1959, Turbulent Transfer in the Lower Atmosphere, Univ. of Chicago Press.

Reiter, E. R. and Lester, P. F.: 1967, 'The Dependence of the Richardson Number on Scale Length', Atmos. Sci. Paper No. 111, Colorado State Univ., Fort Collins, Colorado.

Sasamori, T.: 1970, 'A Numerical Study of Atmospheric and Soil Boundary Layer', J. Atmos. Sci. 27, 1122-1137.

Shir, C. C.: 1972, 'A Numerical Computation of Air Flow over a Sudden Change of Surface Roughness', J. Atmos. Sci. 29, 304-310.

Shir, C. C.: 1973, 'A Preliminary Numerical Study of Atmospheric Turbulent Flows in the Idealized Planetary Boundary Layer', J. Atmos. Sci. 30, 1327-1339.

Stevens, D. W.: 1959, 'Numerical Experiments in Forecasting Air and Soil Temperature Profiles', GRD Note No. 12, Air Force Cambridge Research Lab., Bedford, Mass.

Tag, P. M.: 1969, 'Surface Temperatures in an Urban Environment', M.S. Thesis, Dept. of Meteorol., The Pennsylvania State Univ., Univ. Park. Penn., 69 pp.

Takeuchi, K.: 1961, 'On the Structure of the Turbulent Field in the Surface Boundary Layer', J. Meteorol. Soc. Japan 39, 346-367.

Webb, E. K.: 1970, 'Profile Relationships: The Log-Linear Range and Extension to Strong Stability', Quart. J. Roy. Meteorol. Soc. 96, 67-90.

Wu, S. S.: 1965, 'A Study of Heat Transfer Coefficients in the Lowest 400 meters of the Atmosphere', J. Geophys. Res. 70, 1801-1807.

Wyngaard, J. C.: 1973, 'On Surface Layer Turbulence', in D. A. Haugen (ed.), Workshop on Micrometeorology, Am. Meteorol. Soc., Boston, pp. 101-149.

Wyngaard, J. C., Coté, O. R., and Rao, K. S.: 1974, 'Modeling the Atmospheric Boundary Layer', Adv. Geophys. 18A, 193-212.

Yamamoto, G.: 1959, 'Theory of Turbulent Transfer in Non-Neutral Conditions', J. Meteorol. Soc. Japan 37, 60-70.

Zdundowski, W., Henderson, D., and Hales, J. V.: 1967, 'Prediction of Nocturnal Temperature Changes During a Calm Night', Beiträge Physik Atmosphäre 40, 144-157. 